\title{
Article
}

\section{Synthesis and photophysical properties of meso-aminophenyl-substituted heptamethine dyes as potential leads to new contrast agents}

Okoh, Adeyi Okoh, Critchley, Megan, Bisby, Roger, Lawrence, Clare Louise, Wainwright, Mark and Smith, Robert B

Available at http://clok.uclan.ac.uk/29274/

Okoh, Adeyi Okoh, Critchley, Megan, Bisby, Roger, Lawrence, Clare Louise ORCID: 0000-0003-0170-0079, Wainwright, Mark and Smith, Robert B ORCID: 0000-0002-2829-5360 (2019) Synthesis and photophysical properties of meso-aminophenyl-substituted heptamethine dyes as potential leads to new contrast agents. Coloration Technology, 135 (4). pp. 305-311. ISSN 1472-3581

It is advisable to refer to the publisher's version if you intend to cite from the work. http://dx.doi.org/10.1111/cote.12403

For more information about UCLan's research in this area go to http://www.uclan.ac.uk/researchgroups/ and search for <name of research Group>.

For information about Research generally at UCLan please go to http://www.uclan.ac.uk/research/

All outputs in CLoK are protected by Intellectual Property Rights law, including Copyright law. Copyright, IPR and Moral Rights for the works on this site are retained by the individual authors and/or other copyright owners. Terms and conditions for use of this material are defined in the policies page. 


\section{Coloration Technology}

\section{Synthesis and photophysical properties of meso- aminophenyl substituted heptamethine dyes as potential leads to new contrast agents}

\begin{tabular}{|c|c|}
\hline Journal: & Coloration Technology \\
\hline Manuscript ID & CTE-19-0002.R2 \\
\hline Wiley - Manuscript type: & Full Paper \\
\hline $\begin{array}{r}\text { Date Submitted by the } \\
\text { Author: }\end{array}$ & $\mathrm{n} / \mathrm{a}$ \\
\hline Complete List of Authors: & $\begin{array}{l}\text { Okoh, Okoh Adeyi; University of Central Lancashire, Chemistry, School } \\
\text { of Physical Sciences and Computing } \\
\text { Critchley, Megan; University of Central Lancashire, Chemistry, School of } \\
\text { Physical Sciences and Computing } \\
\text { Bisby, Roger; University of Salford, School of Environment and Life } \\
\text { Sciences } \\
\text { Lawrence, Clare; University of Central Lancashire, School of Pharmacy } \\
\text { and Biomedical Sciences } \\
\text { Wainwright, Mark; Liverpool John Moores University, School of Pharmacy } \\
\text { \& Biomolecular Sciences } \\
\text { Smith, Robert; University of Central Lancashire, Chemistry, School of } \\
\text { Physical Sciences and Computing }\end{array}$ \\
\hline Keywords: & Near Infrared, Stokes Shifts, Heptamethine dyes \\
\hline Abstract: & $\begin{array}{l}\text { The series of rigid meso-aminophenyl substituted heptamethine dyes } \\
\text { presented herein exhibit surprising fluorescence properties, } \\
\text { demonstrating larger Stokes shifts when compared to both structurally- } \\
\text { similar rigid meso-chlorophenyl and linear heptamethine dyes. Based on } \\
\text { their photophysical properties, these are of considerable importance to } \\
\text { the development of contrast agents, within biology and medicine. }\end{array}$ \\
\hline
\end{tabular}

\section{SCHOLARONE Manuscripts}




\title{
Synthesis and photophysical properties of meso-aminophenyl substituted heptamethine dyes as potential leads to new contrast agents
}

\author{
Okoh Adeyi Okoh, ${ }^{a}$ Megan E. Critchley, ${ }^{\text {a }}$ Roger H. Bisby, ${ }^{\mathrm{b}}$ Clare L. Lawrence, ${ }^{\mathrm{c}}$ Mark \\ Wainwright ${ }^{d}$ and Robert B. Smith ${ }^{a^{*}}$ \\ aSchool of Physical Sciences and Computing, University of Central Lancashire, Preston, PR1 \\ $2 \mathrm{HE}, \mathrm{UK}$

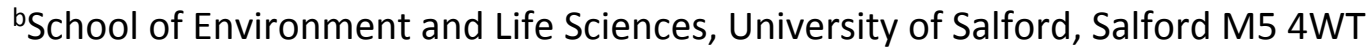 \\ 'School of Pharmacy and Biomedical Sciences, University of Central Lancashire, Preston, PR1 \\ $2 \mathrm{HE}, \mathrm{UK}$ \\ ${ }^{d}$ School of Pharmacy \& Biomolecular Sciences, Liverpool John Moores University, Liverpool \\ L3 $3 \mathrm{AF}$ \\ Corresponding Author: rbsmith@uclan.ac.uk
}

\begin{abstract}
The series of rigid meso-aminophenyl substituted heptamethine dyes presented herein exhibit surprising fluorescence properties, demonstrating larger Stokes shifts when compared to both structurally-similar rigid meso-chlorophenyl and linear heptamethine dyes. Based on their photophysical properties, these are of considerable importance to the development of contrast agents, within biology and medicine.
\end{abstract}

\author{
Submitted to \\ Coloration Technology
}

Full Paper 


\section{Introduction}

The detection, imaging and quantification of biomolecules play a vital part within biomedicine especially in areas relating to the non-invasive monitoring of diseased tissues. ${ }^{1}$ Indeed, the use of fluorescent probes within the biosciences has revolutionised the way biomolecules are visualised. ${ }^{2}$ Fluorescent probes with spectra within the near infrared region (700-1000nm) offer significant advantages over those which absorb within the visible region. Such advantages include minimal tissue auto-fluorescence, resulting mainly from flavins, flavoproteins and NADH, that can often hinder the visualisation of the probe. Other advantages includes, sharper optical contrast with less scatter, deeper light penetration into tissues and the possibility of specific chemotargeting through the attachment of sugars, amino acids, monoclonal antibodies or aptamers, all of which are advantageous for cell and tissue imaging. ${ }^{3}$

The clinically established heptamethine dyes such as Indocyanine Green (ICG) and New Indocyanine Green (IR-820) have proved to be excellent molecular probes; both possess reasonable fluorescent quantum yields, low photo-bleaching, good aqueous solubility, high signal to noise ratio, low toxicity and rapid clearance. ${ }^{4}$ Both probes absorb within the near infrared $800-850 \mathrm{~nm}$ range, which again is advantageous as this is well away from endogenous absorbers such as haemoglobin and melanin. The shift into the near infrared can be attributed to the extension of conjugation due to benzene fusion in the dihydrobenzo[e]indolyl groups which increases the bathochromic shift of these dyes by approximately $30 \mathrm{~nm},{ }^{5}$ in comparison to dyes such as IR-746 and IR-783, neither of which possesses the fused aromatic system. IR820 also has a chloro-substituted cyclohexyl moiety incorporated into the polymethine backbone, allowing for increased photostability. ${ }^{6}$ It also allows the prospect of addition of nucleophilic moieties, which may not only alter the photophysical properties, but also an option of adding groups such as amino acids, oligopeptides or sugars for specific sub-cellular targeting. Such a pathway would be advantageous as discussed above.

Inspired by this, we report the synthesis and photophysical properties for a series of structurally related non-targeting NIR heptamethine cyanine dyes, bearing fused and nonfused benzene subunits. These contain either a polymethine backbone that is partially rigidified by the inclusion of a central cyclic moiety with a chlorine atom in the meso position, or alternatively an aminophenyl moiety at the meso position. Our intention was to compare the photophysical properties (absorption and fluorescence spectra, fluorescence quantum yields and Stokes shifts) of the dyes bearing an aniline moiety against those bearing a chlorine atom at the meso position. These were also compared to the simple linear heptamethine conjugated chain analogues, in order to investigate the effects of replacing the meso-chlorine atom with a phenylamino moiety, in addition to determining the effect of the fused benzene ring on the dyes' absorption characteristics. 


\section{Experimental}

\section{General information}

NMR spectra were recorded on either a Bruker DPX 250 MHz, Bruker Avance-III 300 MHz or a Bruker Avance $400 \mathrm{MHz}$ spectrometer at ambient temperature. Chemical shifts are reported

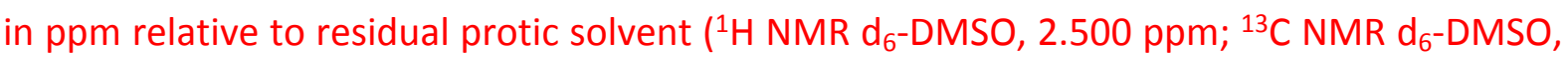
39.520 ppm). Low and high resolution mass spectra (HRMS) were obtained using electrospray ionization (ESI) mass spectrometry on a hybrid linear ion trap-fourier transform mass spectrometer. Accurate mass measurements were carried out for novel compounds by the EPSRC National mass spectrometry service research group using the Thermofisher LTQ Orbitrap XL. Stock solutions for UV-Vis spectroscopy of dyes were prepared in methanol. The absorbance and fluorescence spectra of each of the dyes were measured sequentially to reduce photobleaching and solubility issues. The fluorescence quantum yields of the dyes were calculated using the relative method i.e. integrated fluorescence peak area versus fraction of light absorbed at the excitation wavelength were plotted for both the standards and cyanine dyes. ${ }^{1}$ Fluorescence quantum yields $(\boldsymbol{\phi})$ were measured using a fluorimeter based on an Innovative Photonic Solutions $785 \mathrm{~nm}$ diode laser, operating with a power output of $8 \mathrm{~mW}$, an Andor Shamrock SR-303i spectrograph and an Andor iDus CCD detector (model DU420A-BR-DD). Fluorescence was detected at right angles without any filters. The spectral response of the system was corrected following the method outlined by Kosch and coworkers. ${ }^{2}$ Absorbance values (A) of solutions at $785 \mathrm{~nm}$ were measured using a Perkin Elmer Lambda 25 spectrophotometer. Corrected fluorescence spectra for a series of solutions of increasing concentration were rescaled in energy and the integrated intensity was plotted versus $1-10^{-A}$, with $A(785 \mathrm{~nm}) \leq 0.1$. The quantum yields were obtained from the relative slopes of such plots compared with that from solutions of ICG in dimethyl sulfoxide. For determinations in different solvents, the refractive index $\left(\mathrm{n}^{2}\right)$ correction was applied. Thin Layer Chromatography (TLC) was carried out on Machery-Nagel polygramSil/G/UV254 precoated plates. Melting point (m.p) analysis was carried out using a Griffin melting point apparatus. Infra-red spectra (1800-800 $\left.\mathrm{cm}^{-1}\right)$ were recorded on a Perkin Elmer Spectrum RX 1 with a Specac Golden Gate ${ }^{\mathrm{TM}}$ ATR accessory and values are quoted in wavenumbers. All chemicals were purchased from commercial sources and used without further purification. Purification of the final dyes were accomplished via column chromatography on Silca gel eluting with the solvent blends listed within the experimental methods as well as the supporting information.

The synthesis of a $\mathbf{1 e}, \mathbf{2} \mathbf{e}$ and the reactive imine from the Vilsmeier-Haack formylation of cyclohexanone (VMI) are shown in the experimental section below for clarity. More information concerning the synthesis of these dyes, the reactive pyridinium salt and the linear hepthamethine dyes (4a-j) is shown in Supporting Information. The full synthesis of compounds $3 a-3 j$ is given below. 


\section{1-Benzyl-2,3,3-trimethyl-3H-indol-1-ium bromide (1e)}

To a refluxing solution of benzyl bromide $(0.80 \mathrm{~mL}, 6.74 \mathrm{mmol})$ in acetonitrile $(20.0 \mathrm{~mL})$ was added dropwise 2,3,3-trimethylindolenine $(1.00 \mathrm{~g}, 6.28 \mathrm{mmol})$ in acetonitrile $(20.0 \mathrm{~mL})$ and the reaction was held at reflux with constant stirring for 2 days. Upon cooling, the precipitate produced was isolated at the pump, washed with $n$-hexane and dried in vacuo to give the product 1e (1.65 $\mathrm{g}, 80 \%)$ as a red hygroscopic solid.

${ }^{1} \mathrm{H}$ NMR ( $\mathrm{d}_{6}$-DMSO, $250 \mathrm{MHz}$ ) $\delta 7.89$ (d, J=7.0 Hz, 1H, Ar-H), 7.84 (d, J=7.0 Hz, 1H, Ar-H), 7.67$7.59(\mathrm{~m}, 2 \mathrm{H}, \mathrm{Ar}-\mathrm{H}), 7.45-7.38(\mathrm{~m}, 5 \mathrm{H}, \mathrm{Ar}-\mathrm{H}), 5.87$ (s, 2H, N-CH ), 3.02(s, 3H, N-C-CH $), 1.55$ (s, $\left.6 \mathrm{H} \mathrm{C}-\left(\mathrm{CH}_{3}\right)_{2}\right) .{ }^{13} \mathrm{C}$ NMR $\left(\mathrm{d}_{6}\right.$-DMSO, $\left.62.8 \mathrm{MHz}\right) \delta 198.7,142.5,141.6,132.7,130.1,129.5,129.3$, 128.1, 124.2, 116.5, 55.1, 51.2, 22.7, 15.1, 9.8. IR (ATR) 2969, 1603, 1454, 931, 741, 701, 567 $\mathrm{cm}^{-1}$. MS (ESI) m/z: $250[\mathrm{M}]^{+}$.

\section{$N$-[5-Anilino-3-chloro-2,4-(propane-1,3-diyl)-2,4-pentadiene]anilinium chloride (VMI)}

To a stirred solution of anhydrous DMF $(13.0 \mathrm{~mL}, 168 \mathrm{mmol})$ held at $0^{\circ} \mathrm{C}$ was added dropwise $\mathrm{POCl}_{3}(11.0 \mathrm{~mL}, 117 \mathrm{mmol})$. After $30 \mathrm{~min}$, cyclohexanone $(5.50 \mathrm{~mL}, 53.1 \mathrm{mmol})$ was added and

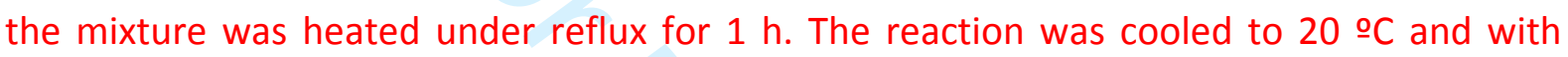
constant stirring, a mixture of aniline/EtOH [1:1 (v/v), $18.0 \mathrm{~mL}]$ was added dropwise. The reaction was continued for $30 \mathrm{~min}$ at $20 \stackrel{\circ}{ } \mathrm{C}$ with vigorous stirring and the deep purple solution was poured into $\mathrm{H}_{2} \mathrm{O} / \mathrm{HCl}$ [10:1 (v/v), $110 \mathrm{~mL}$ ]. After two hours of the solution being held in an ice bath, the crystals formed were isolated at the pump and washed with cold $\mathrm{H}_{2} \mathrm{O}$ and $\mathrm{Et}_{2} \mathrm{O}$. The solid was allowed to dry at the pump to yield $\mathrm{N}$-[5-anilino-3-chloro-2,4-(propane1,3-diyl)-2,4-pentadiene]anilinium chloride (15.4 g, 81\%) as a dark purple solid.

${ }^{1} \mathrm{H}$ NMR ( $\mathrm{d}_{6}$-DMSO, $250 \mathrm{MHz}$ ) $\delta 11.2$ (br s, 2H, NH), 8.55 (d, J=14.0 Hz, 2H, CHalkene), 7.68 (d, $J=7.0 \mathrm{~Hz}, 4 \mathrm{H}, \mathrm{Ar}-\mathrm{H}), 7.44(\mathrm{t}, J=7.0 \mathrm{~Hz}, 4 \mathrm{H}, \mathrm{Ar}-\mathrm{H}), 7.30-7.28(\mathrm{~m}, 2 \mathrm{H}, \mathrm{Ar}-\mathrm{H}), 3.03(\mathrm{t}, J=6.0 \mathrm{~Hz}, 4 \mathrm{H}$, $\left.\mathrm{CH}_{2}-\mathrm{CH}_{2}\right), 2.05-1.98\left(\mathrm{~m}, 2 \mathrm{H}, \mathrm{CH}_{2}-\mathrm{CH}_{2}\right)$. IR (ATR) 1609, 1562, 1457, 1267, 1179, 752, 683, 565 $\mathrm{cm}-1 . \mathrm{MS}(\mathrm{ESI}) \mathrm{m} / \mathrm{z}: 323.22[\mathrm{M}]^{+}$.

\section{1-Benzyl-2-[(E)-2-[(3E)-3-\{2-[(2E)-1-benzyl-3,3-dimethyl-2,3-dihydro-1H-indol-2- ylidene]ethylidene\}-2-chlorocyclohex-1-en-1-yl]ethenyl]-3,3-dimethyl-3H-indol-1-ium bromide (2e).}

To a solution of $1 \mathrm{e}(0.66 \mathrm{~g}, 2 \mathrm{mmol})$ and anhydrous sodium acetate $(0.16 \mathrm{~g}, 1.95 \mathrm{mmol})$ in EtOH (30.0 $\mathrm{mL}) \quad$ was added $N$-[5-anilino-3-chloro-2,4-(propane-1,3-diyl)-2,4pentadiene]anilinium chloride $(0.36 \mathrm{~g}, 1 \mathrm{mmol})$ with constant stirring, and the solution was heated under reflux for $4 \mathrm{~h}$. Upon cooling, the solution was evaporated to dryness under reduced pressure and the crude product was purified by column chromatography on silica gel eluting with $\mathrm{CHCl}_{3}$ 9: $\mathrm{MeOH} 1$ to obtain $2 \mathrm{e}(0.10 \mathrm{~g}, 15 \%)$ as a shiny purple solid.

${ }^{1} \mathrm{H}$ NMR ( $\mathrm{d}_{6}$-DMSO, $400 \mathrm{MHz}$ ) $\delta 8.25$ (d, J=14.0 Hz, 2H, CHalkene), 7.41- $7.28(\mathrm{~m}, 18 \mathrm{H}, \mathrm{Ar}-\mathrm{H}), 6.35$ (d, J=14.0 Hz, 2H, CH alkene), 4.95 (br s, 4H, N-CH $-\mathrm{Ph}), 2.50-2.45\left(\mathrm{~m}, 4 \mathrm{H}, \mathrm{CH}_{2}-\mathrm{CH}_{2}\right), 1.69(\mathrm{~s}, 12 \mathrm{H}$, $\left.\mathrm{C}^{-} \mathrm{CH}_{3}\right), 1.30-1.26\left(\mathrm{~m}, 2 \mathrm{H}, \mathrm{CH}_{2}-\mathrm{CH}_{2}-\mathrm{CH}_{2}\right) . \mathrm{IR}$ (ATR) 1543, 1363, 1225, 1099, 896, 784, 668, 597 $\mathrm{cm}^{-1}$. MS (ESI) m/z: $635[\mathrm{M}]^{+} . \lambda_{\max }=760 \mathrm{~nm}$. 
1,3,3-Trimethyl-2-[(E)-2-[(3E)-2-(phenylamino)-3-\{2-[(2E)-1,3,3-trimethyl-2,3-dihydro-1Hindol-2-ylidene]ethylidene\}cyclohex-1-en-1-yl]ethenyl]-3H-indol-1-ium iodide (3a)

Aniline $(1.00 \mathrm{mmol})$ was added by syringe to a solution of $2 \mathrm{a}(0.10 \mathrm{mmol})$ dissolved in anhydrous DMF (10.0 mL) under nitrogen. The reaction mixture was stirred at $85^{\circ} \mathrm{C}$ overnight under nitrogen. The solvent was removed under reduced pressure, the crude product was purified by column chromatography on silica gel eluting with DCM 9 : $\mathrm{MeOH} 1$, then and treated with propan-2-ol to afford 3 a (20 mg, 30\%) as a dark green solid.

${ }^{1} \mathrm{H}$ NMR ( $\mathrm{d}_{6}$-DMSO, $\left.250 \mathrm{MHz}\right) \delta 8.62$ (br s, 1H, Ar-NH), 7.93 (d, J=14.0 Hz, 2H, CH alkene), 7.49 (d, J=6.0 Hz, 2H, Ar-H), 7.30-7.21 (m, 9H, Ar-H), 6.90 (d, J=6.0 Hz, 2H, ArH), 6.05 (d, J=14.0 Hz, $\left.2 \mathrm{H}, \mathrm{CH}_{\text {alkene }}\right), 3.65\left(\mathrm{~s}, 6 \mathrm{H}, \mathrm{N}-\mathrm{CH}_{3}\right), 2.62\left(\mathrm{t}, \mathrm{J}=6.0 \mathrm{~Hz}, 4 \mathrm{H}, \mathrm{CH}_{2}\right), 1.85-1.79\left(\mathrm{~m}, 2 \mathrm{H}, \mathrm{CH}_{2}\right), 1.03$ (s, $12 \mathrm{H}, \mathrm{C}-\mathrm{CH}_{3}$ ). IR (ATR) 2928, 1555, 1484, 1436, 1349, 1308, 1206, 1149, 915, $786 \mathrm{~cm}^{-1}$. MS (ESI) $\mathrm{m} / \mathrm{z}: 540[\mathrm{M}]^{+} \cdot \lambda_{\max }=736$ nm. m.p. $213-215^{\circ} \mathrm{C}$.

1-Ethyl-2-[(E)-2-[(3E)-3-\{2-[(2E)-1-ethyl-3,3-dimethyl-2,3-dihydro-1H-indol-2ylidene]ethylidene\}-2-(phenylamino)cyclohex-1-en-1-yl]ethenyl]-3,3-dimethyl-3H-indol-1ium iodide (3b).

$\mathbf{3 b}$ was synthesised as for $\mathbf{3 a}$ using $\mathbf{2} \mathbf{b}(0.10 \mathrm{mmol})$, aniline $(1.00 \mathrm{mmol})$, and anhydrous DMF $(10.0 \mathrm{~mL})$. The solvent was removed under reduced pressure, the crude product was purified by column chromatography on silica gel eluting with DCM 9 : MeOH 1 to afford 3 b $(24 \mathrm{mg}$, $35 \%)$ as a shiny dark blue solid.

${ }^{1} \mathrm{H} \mathrm{NMR}\left(\mathrm{CDCl}_{3}, 400 \mathrm{MHz}\right) \delta 9.05$ (br s, $\left.1 \mathrm{H}, \mathrm{Ar}-\mathrm{NH}\right), 8.16$ (d, J=14.0 Hz, 2H, $\left.\mathrm{CH}_{\text {alkene }}\right), 7.41-7.35$ (m, 5H, ArH), 7.22 (t, J=8.0 Hz, 4H, Ar-H), 7.09 (t, J=7.0 Hz, 2H, Ar-H), 6.92 (d, J=8.0 Hz, 2H, Ar$\mathrm{H}), 6.80$ (t, J=7.0 Hz, 2H, Ar-H), 5.79 (d, J=14.0 Hz, 2H, CHalkene), 3.56 (q, J=9.0 Hz, 4H, NCH $\mathrm{NC}_{2}$, $2.57\left(\mathrm{t}, J=6.0 \mathrm{~Hz}, 4 \mathrm{H}, \mathrm{CH}_{2}\right), 1.92-1.84\left(\mathrm{~m}, 2 \mathrm{H}, \mathrm{CH}_{2}\right), 1.72\left(\mathrm{t}, \mathrm{J}=6.0 \mathrm{~Hz}, 6 \mathrm{H}, \mathrm{CH}_{3}\right), 1.36(\mathrm{~s}, 12 \mathrm{H}, \mathrm{C}-$ $\mathrm{CH}_{3}$ ). IR (ATR) 2922, 1560, 1484, 1436, 1342, 1318, 1206, 1129, 905, $783 \mathrm{~cm}^{-1}$. MS (ESI) m/z $568[\mathrm{M}]^{+} . \lambda_{\max }=735$ nm. m.p. $217-219^{\circ} \mathrm{C}$.

\section{2-[(E)-2-[(3E)-3-\{2-[(2E)-3,3-Dimethyl-1-propyl-2,3-dihydro-1H-indol-2-ylidene]ethylidene\}-} 2-(phenylamino)cyclohex-1-en-1-yl]ethenyl]-3,3-dimethyl-1-propyl-3H-indol-1-ium iodide (3c).

$3 \mathbf{c}$ was synthesised as for $\mathbf{3 a}$ using $\mathbf{2 c}(0.10 \mathrm{mmol})$, aniline $(1.00 \mathrm{mmol})$ and anhydrous DMF $(10.0 \mathrm{~mL})$. The solvent was removed under reduced pressure, the crude product was purified by column chromatography on silica gel eluting with DCM 9 : MeOH 1 to afford 3c (31 mg, $43 \%)$ as a shiny dark blue solid.

${ }^{1} \mathrm{H}$ NMR $\left(\mathrm{CDCl}_{3}, 400 \mathrm{MHz}\right) \delta 9.02$ (br s, $\left.1 \mathrm{H}, \mathrm{Ar}-\mathrm{NH}\right), 8.14$ (d, J=14.0 Hz, 2H, $\left.\mathrm{CH}_{\text {alkene }}\right), 7.45-7.38$ (m, 3H, ArH), 7.18-7.12 (m, 5H, Ar-H), 7.09 (t, J=7.0 Hz, 2H, Ar-H), 6.90 (d, J=8.0 Hz, 2H, Ar-H), 6.78 (t, J=7.0 Hz, 1H, Ar-H), 5.79 (d, J=14.0 Hz, 2H, CHalkene), 3.88 (t, J=7.0 Hz, 4H, NCH ), 2.56 (t, J=6.0 Hz, 4H, CH $\left.\mathrm{CH}_{2}\right), 1.93$ (quin, J=6.0 Hz, 2H, $\left.\mathrm{CH}_{2}\right), 1.83-1.76\left(\mathrm{~m}, 4 \mathrm{H}, \mathrm{CH}_{2}\right), 1.38(\mathrm{~s}, 12 \mathrm{H}, \mathrm{C}-$ $\mathrm{CH}_{3}$ ), 1.01 (t, J=7.0 Hz, 6H, CH $2-\mathrm{CH}_{3}$ ). IR (ATR) 2920, 1549, 1431, 1344, 1232, 1151, 944, 787 $\mathrm{cm}^{-1}$. MS (ESI) m/z: $596[\mathrm{M}]^{+} . \lambda_{\max }=746$ nm. m.p. $215-217^{\circ} \mathrm{C}$. 


\section{2-[(E)-2-[(3E)-3-\{2-[(2E)-3,3-Dimethyl-1-(4-sulfonatobutyl)-2,3-dihydro-1H-indol-2- ylidene]ethylidene\}-2-(phenylamino)cyclohex-1-en-1-yl]ethenyl]-3,3-dimethyl-1-(4- sulfonatobutyl)-3H-indol-1-ium (3d).}

$\mathbf{3 d}$ was synthesised as for $3 \mathbf{a}$ using $\mathbf{2 a}(0.10 \mathrm{mmol})$, aniline $(1.00 \mathrm{mmol})$ and anhydrous DMF $(10.0 \mathrm{~mL})$. The solvent was removed under reduced pressure, the crude product was purified by column chromatography on silica gel eluting with $\mathrm{CHCl}_{3} 8: \mathrm{MeOH} 2$ to afford $\mathbf{3 d}(31 \mathrm{mg}$, $40 \%)$ as a dark green solid.

${ }^{1} \mathrm{H}$ NMR (d ${ }_{6}$-DMSO, $\left.400 \mathrm{MHz}\right) \delta 8.65$ (br s, 1H, Ar-NH), 7.98 (d, J=14.0 Hz, 2H, CH alkene), 7.43 (d, J=7.0 Hz, 2H, ArH), 7.33-7.28 (m, 4H, Ar-H), 7.22 (t, J=8.0 Hz, 2H, Ar-H), 7.13-6.99 (m, 2H, $\operatorname{Ar}-\mathrm{H}), 6.93(\mathrm{~d}, J=7.0 \mathrm{~Hz}, 2 \mathrm{H}, \mathrm{Ar}-\mathrm{H}), 6.71(\mathrm{~d}, J=7.0 \mathrm{~Hz}, 1 \mathrm{H}, \mathrm{Ar}-\mathrm{H}), 6.10$ (d, J=14.0 Hz, 2H, CH alkene),

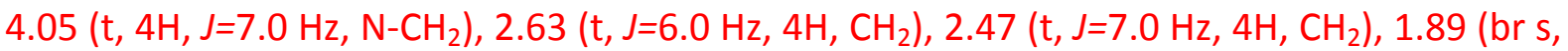
$\left.2 \mathrm{H}, \mathrm{CH}_{2}\right), 1.71-1.63\left(\mathrm{~m}, 8 \mathrm{H}, \mathrm{CH}_{2}\right), 1.28$ (s, 12H, C-CH $\mathrm{CH}_{3} . \mathrm{IR}$ (ATR) 2928, 1508, 1437, 1366, 1097, $1101,908,790 \mathrm{~cm}^{-1}$. MS (ESI) m/z: $784[\mathrm{M}+2 \mathrm{H}]^{+} . \lambda_{\max }=745 \mathrm{~nm}$. m.p. $179-181^{\circ} \mathrm{C}$.

\section{1-Benzyl-2-[(E)-2-[(3E)-3-\{2-[(2E)-1-benzyl-3,3-dimethyl-2,3-dihydro-1H-indol-2-}

ylidene]ethylidene\}-2-(phenylamino)cyclohex-1-en-1-yl]ethenyl]-3,3-dimethyl-3H-indol1ium bromide (3e).

3e was synthesised as for $3 a$ using 2 e $(0.10 \mathrm{mmol})$, aniline $(1.00 \mathrm{mmol})$ and anhydrous DMF $(10.0 \mathrm{~mL})$. The solvent was removed under reduced pressure, the crude product was purified by column chromatography on silica gel eluting $\mathrm{CHCl}_{3} 9: \mathrm{MeOH} 1$ to afford $3 e(13 \mathrm{mg}, 17 \%)$ as a dark shiny blue solid.

${ }^{1} \mathrm{H}$ NMR ( $\mathrm{d}_{6}$-DMSO, $\left.400 \mathrm{MHz}\right) \delta 8.87$ (br s, 1H, Ar-NH), 7.95 (d, J=14.0 Hz, 2H, CHalkene), 7.48 (d, J=7.0 Hz, 2H, ArH), 7.33-7.32 (m, 4H, Ar-H), 7.31-7.28 (m, 6H, Ar-H), 7.22-7.19 (m, 6H, ArH), 7.14 (t, J=7.0 Hz, 2H, Ar-H), 6.95 (d, J=7.0 Hz, 2H, Ar-H), 6.78 (t, J=7.0 Hz, 1H, Ar-H), 6.07

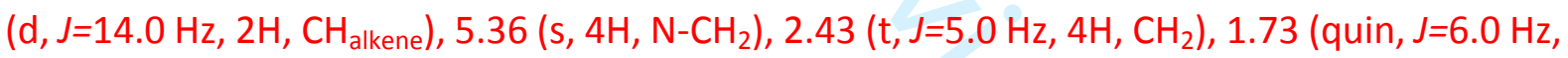
$2 \mathrm{H}, \mathrm{CH}_{2}$ ), 1.33 (s, 12H, CH ). IR (ATR) 2921, 1504, 1436, 1361, 1224, 1005, 897, $787 \mathrm{~cm}^{-1}$. MS (ESI) $\mathrm{m} / \mathrm{z}: 692[\mathrm{M}]^{+}$. HRMS(ESI): Calcd for $\mathrm{C}_{50} \mathrm{H}_{52} \mathrm{~N}_{3}[\mathrm{M}]^{+}$692.3978, found 692.3980. $\lambda_{\max }=736$ nm. m.p. $210-212^{\circ} \mathrm{C}$.

\section{1,1,3-Trimethyl-2-[(E)-2-[(3E)-2-(phenylamino)-3-\{2-[(2E)-1,1,3-trimethyl-1H,2H,3H-} benzo[ $e]$ indol-2-ylidene]ethylidene\}cyclohex-1-en-1-yl]ethenyl]-1H-benzo[e]indol-3-ium iodide (3f).

3f was synthesised as for $\mathbf{3 a}$ using $\mathbf{2} \mathbf{f}(0.10 \mathrm{mmol})$, aniline $(1.00 \mathrm{mmol})$ and anhydrous DMF $(10.0 \mathrm{~mL})$. The solvent was removed under reduced pressure, the crude product was purified by column chromatography on silica gel eluting with DCM 9 : $\mathrm{MeOH} 1$ to afford $3 f(34 \mathrm{mg}$, $44 \%)$ as a shiny dark blue solid.

${ }^{1} \mathrm{H}$ NMR (d 6 -DMSO, $300 \mathrm{MHz}$ ) $\delta 8.76$ (br s, $\left.1 \mathrm{H}, \mathrm{NH}\right), 8.37$ (t, J=9.0 Hz, 2H, Ar-H), 8.16 (d, J=9.0 $\mathrm{Hz}, 2 \mathrm{H}, \mathrm{Ar}-\mathrm{H}), 7.77-7.67(\mathrm{~m}, 4 \mathrm{H}, \mathrm{Ar}-\mathrm{H}), 7.66-7.63(\mathrm{~m}, 5 \mathrm{H}, \mathrm{Ar}-\mathrm{H}), 7.46$ (t, J=9.0 Hz, 2H, Ar-H), 7.32 (t, J=9.0 Hz, 1H, Ar-H), 7.25 (t, J=9.0 Hz, 2H, Ar-H), 6.59 (d, J=12.0 Hz, 2H, CH alkene), 5.42 (d, J=12.0 Hz, $\left.2 \mathrm{H}, \mathrm{CH}_{\text {alkene }}\right), 3.70\left(\mathrm{~s}, 6 \mathrm{H}, \mathrm{N}-\mathrm{CH}_{3}\right), 2.83-2.66\left(\mathrm{~m}, 2 \mathrm{H}, \mathrm{CH}_{2}\right), 2.28(\mathrm{t}, J=6.0 \mathrm{~Hz}, 4 \mathrm{H}$, $\mathrm{CH}_{2}$ ), 1.20 (s, 12H, $\mathrm{CH}_{3}$ ). IR (ATR) 2992, 1515, 1441, 1350, 1228, 1011, 929, $747 \mathrm{~cm}^{-1}$. MS (ESI) 
m/z: $638[\mathrm{M}]^{+}$. HRMS(ESI): Calcd for $\mathrm{C}_{46} \mathrm{H}_{46} \mathrm{~N}_{3}[\mathrm{M}]^{+} 638.3530$, found 638.3529. $\lambda_{\max }=780 \mathrm{~nm}$. m.p. $188-190^{\circ} \mathrm{C}$.

\section{3-Ethyl-2-[(E)-2-[(3E)-3-\{2-[(2E)-3-ethyl-1,1-dimethyl-1H,2H,3H-benzo[e]indol-2- ylidene]ethylidene\}-2-(phenylamino)cyclohex-1-en-1-yl]ethenyl]-1,1-dimethyl-1H- benzo[e]indol-3-ium iodide $(3 \mathrm{~g})$.}

$\mathbf{3 g}$ was synthesised as for $3 \mathrm{a}$ using $\mathbf{2} \mathbf{g}(0.10 \mathrm{mmol})$, aniline $(1.00 \mathrm{mmol})$ and anhydrous DMF $(10.0 \mathrm{~mL})$. The solvent was removed under reduced pressure, the crude product was purified by column chromatography on silica gel eluting with DCM 9 : MeOH 1 to afford 3 g $(27$ mg, $34 \%)$ as a shiny dark blue solid.

${ }^{1} \mathrm{H}$ NMR (d ${ }_{6}$-DMSO, $300 \mathrm{MHz}$ ) $\delta 8.74$ (br s, $\left.1 \mathrm{H}, \mathrm{NH}\right), 8.14-8.01(\mathrm{~m}, 8 \mathrm{H}, \mathrm{Ar}-\mathrm{H}), 7.67(\mathrm{~d}, J=9.0 \mathrm{~Hz}$, $2 \mathrm{H}, \operatorname{Ar}-\mathrm{H}$ ), 7.60 (t, J=9.0 Hz, 2H, Ar-H), 7.47 (t, J=9.0 Hz, 2H, Ar-H), 7.31 (d, J=15.0 Hz, 2H, $\left.\mathrm{CH}_{\text {alkene }}\right), 7.03$ (d, J=9.0 Hz, 2H, Ar-H), 6.79 (t, J=6.0 Hz, $\left.1 \mathrm{H}, \mathrm{Ar}-\mathrm{H}\right), 6.14$ (d, J= $15.0 \mathrm{~Hz}, 2 \mathrm{H}$, $\left.\mathrm{CH}_{\text {alkene }}\right), 4.25\left(\mathrm{q}, \mathrm{J}=6.0 \mathrm{~Hz}, 4 \mathrm{H}, \mathrm{N}-\mathrm{CH}_{2}\right), 2.70\left(\mathrm{t}, \mathrm{J}=6.0 \mathrm{~Hz}, 4 \mathrm{H}, \mathrm{CH}_{2}\right), 1.96-1.87\left(\mathrm{~m}, 4 \mathrm{H}, \mathrm{CH}_{2}\right), 1.62$ (s, 12H, CH3), 1.32 (t, J=9.0 Hz, 6H, CH3). IR (ATR) 2921, 1546, 1430, 1346, 1226, 1001, 901, $\mathrm{cm}^{-1}$. MS (ESI) m/z: $668[\mathrm{M}]^{+}$. HRMS(ESI): Calcd for $\mathrm{C}_{48} \mathrm{H}_{50} \mathrm{~N}_{3}[\mathrm{M}]^{+} 668.3999$, found 668.3993. $\lambda_{\max }=776$ nm. m.p. $188-190^{\circ} \mathrm{C}$.

\section{2-[(E)-2-[(3E)-3-\{2-[(2E)-1,1-imethyl-3-propyl-1H,2H,3H-benzo[e]indol-2-} ylidene]ethylidene\}-2-(phenylamino)cyclohex-1-en-1-yl]ethenyl]-1,1-dimethyl-3-propyl$1 \mathrm{H}$-benzo[e]indol-3-ium iodide (3h).

$3 \mathrm{~h}$ was synthesised as for $\mathbf{3 a}$ using $\mathbf{2} \mathbf{h}(0.10 \mathrm{mmol})$, aniline $(1.00 \mathrm{mmol})$ and anhydrous DMF $(10.0 \mathrm{~mL})$. The solvent was removed under reduced pressure, the crude product was purified by column chromatography on silica gel eluting with DCM 9 : MeOH 1 to afford $3 \mathrm{~h}(31 \mathrm{mg}$, $38 \%$ ) as a shiny dark blue solid.

${ }^{1} \mathrm{H}$ NMR $\left(\mathrm{d}_{6}\right.$-DMSO, $\left.300 \mathrm{MHz}\right) \delta 8.76(\mathrm{br} \mathrm{s}, 1 \mathrm{H}, \mathrm{NH}), 8.13-8.07(\mathrm{~m}, 4 \mathrm{H}, \mathrm{Ar}-\mathrm{H}), 8.02-7.98(\mathrm{~m}, 4 \mathrm{H}$, Ar-H), 7.68 (d, J=9.0 Hz, 2H, Ar-H), 7.57 (t, J=9.0 Hz, 2H, Ar-H), 7.46 (t, J=9.0 Hz, 2H, Ar-H), 7.31 (d, J=15.0 Hz, 2H, CH alkene), 7.03 (d, J=9.0 Hz, 2H, Ar-H), 6.78 (t, J=6.0 Hz, 1H, Ar-H), 6.14 (d, J= $\left.15.0 \mathrm{~Hz}, 2 \mathrm{H}, \mathrm{CH}_{\text {alkene }}\right), 4.20\left(\mathrm{t}, \mathrm{J}=9.0 \mathrm{~Hz}, 4 \mathrm{H}, \mathrm{N}-\mathrm{CH}_{2}\right), 2.68\left(\mathrm{t}, \mathrm{J}=6.0 \mathrm{~Hz}, 4 \mathrm{H}, \mathrm{CH}_{2}\right), 1.92-180(\mathrm{~m}, 2 \mathrm{H}$, $\left.\mathrm{CH}_{2}\right), 1.78-1.61\left(\mathrm{~m}, 4 \mathrm{H}, \mathrm{N}-\mathrm{CH}_{2}\right), 0.97\left(\mathrm{~s}, 12 \mathrm{H}, \mathrm{CH}_{3}\right), 0.97\left(\mathrm{t}, \mathrm{J}=9.0 \mathrm{~Hz}, 6 \mathrm{H}, \mathrm{CH}_{3}\right)$. IR (ATR) 2922, 1547, 1430, 1342, 1225, 1001, 897, $711 \mathrm{~cm}^{-1}$. MS (ESI) m/z: 696 [M] ${ }^{+}$. HRMS(FAB): Calcd for $\mathrm{C}_{50} \mathrm{H}_{54} \mathrm{~N}_{3}[\mathrm{M}]^{+} 696.4312$, found $696.4304 . \lambda_{\max }=778$ nm. m.p. $188-190^{\circ} \mathrm{C}$.

\section{2-[(E)-2-[(3E)-3-\{2-[(2E)-1,1-Dimethyl-3-(4-sulfonatobutyl)-1H,2H,3H-benzo[e]indol-2-} ylidene]ethylidene\}-2-(phenylamino)cyclohex-1-en-1-yl]ethenyl]-1,1-dimethyl-3-(4sulfonatobutyl)-1H-benzo[e]indol-3-ium (3i).

$3 \mathbf{i}$ was synthesised as for $\mathbf{3} \mathbf{a}$ using $\mathbf{2} \mathbf{i}(0.10 \mathrm{mmol})$, aniline $(1.00 \mathrm{mmol})$, and anhydrous DMF $(10.0 \mathrm{~mL})$. The solvent was removed under reduced pressure, the crude product was purified by column chromatography on silica gel eluting with DCM 9 : MeOH 1 to afford $3 \mathbf{i}$ (35 mg, $40 \%)$ as a green solid. 


\begin{abstract}
${ }^{1} \mathrm{H}$ NMR (d ${ }_{6}$-DMSO, $\left.300 \mathrm{MHz}\right) \delta 8.75$ (brs, $\left.1 \mathrm{H}, \mathrm{NH}\right), 8.12-8.08(\mathrm{~m}, 4 \mathrm{H}, \mathrm{Ar}-\mathrm{H}), 8.00(\mathrm{~d}, J=6.0 \mathrm{~Hz}$, $4 \mathrm{H}, \mathrm{Ar}-\mathrm{H}$ ), 7.69 (d, J=9.0 Hz, 2H, Ar-H), 7.56 (t, J=15.0 Hz, 2H, CHalkene), 7.45 (t, J=9.0 Hz, 2H, ArH), 7.30 (t, J=6.0 Hz, 2H, Ar-H), $7.03(\mathrm{~d}, J=9.0 \mathrm{~Hz}, 2 \mathrm{H}, \mathrm{Ar}-\mathrm{H}), 6.77(\mathrm{t}, J=6.0 \mathrm{~Hz}, 1 \mathrm{H}, \mathrm{Ar}-\mathrm{H}), 6.16$ (d, J= $\left.15.0 \mathrm{~Hz}, 2 \mathrm{H}, \mathrm{CH}_{\text {alkene }}\right), 4.19\left(\mathrm{t}, J=6.0 \mathrm{~Hz}, 4 \mathrm{H}, \mathrm{N}-\mathrm{CH}_{2}\right), 2.73\left(\mathrm{t}, J=6.0 \mathrm{~Hz}, 4 \mathrm{H}, \mathrm{CH}_{2}\right), 2.48-2.35$ $\left(\mathrm{m}, 4 \mathrm{H}, \mathrm{CH}_{2}\right), 1.93-187\left(\mathrm{~m}, 2 \mathrm{H}, \mathrm{CH}_{2}\right), 1.76-1.65\left(\mathrm{~m}, 8 \mathrm{H}, \mathrm{CH}_{2}\right), 1.60\left(\mathrm{~s}, 12 \mathrm{H}, \mathrm{CH}_{3}\right)$. IR (ATR) 2928, $1594,1433,1349,1227,1004,886,714 \mathrm{~cm}^{-1}$. MS (ESI) m/z: $884[\mathrm{M}]^{+} . \lambda_{\max }=779$ nm. m.p. $230-$ $232^{\circ} \mathrm{C}$.
\end{abstract}

3-Benzyl-2-[(E)-2-[(3E)-3-\{2-[(2E)-3-benzyl-1,1-dimethyl-1H,2H,3H-benzo[e]indol-2ylidene]ethylidene\}-2-(phenylamino)cyclohex-1-en-1-yl]ethenyl]-1,1-dimethyl-1Hbenzo[ $e$ ]indol-3-ium bromide (3j).

3j was synthesised as for $\mathbf{3 a}$ using $\mathbf{2} \mathbf{j}(0.10 \mathrm{mmol})$, aniline $(1.00 \mathrm{mmol}$ and anhydrous DMF $(10.0 \mathrm{~mL})$. The solvent was removed under reduced pressure, the crude product was purified by column chromatography on silica gel eluting with DCM 9 : MeOH 1 to afford 3j $(31 \mathrm{mg}$, $34 \%)$ as a shiny dark blue solid.

${ }^{1} \mathrm{H}$ NMR (d ${ }_{6}$-DMSO, $300 \mathrm{MHz}$ ) $\delta 8.93$ (brs, $1 \mathrm{H}, \mathrm{NH}$ ), 8.32 (s, 1H, Ar-H), 8.16 (d, J=9.0 Hz, 2H, Ar$\mathrm{H}), 8.08$ (d, J=15.0 Hz, 2H, CHalkene), 7.99-7.90 (m, 4H, Ar-H), 7.62-7.33 (m, 4H, Ar-H), 7.46 (t, $J=9.0 \mathrm{~Hz}, 2 \mathrm{H}, \mathrm{Ar}-\mathrm{H}), 7.31-7.23(\mathrm{~m}, 11 \mathrm{H}, \mathrm{Ar}-\mathrm{H}), 7.03(\mathrm{~d}, J=9.0 \mathrm{~Hz}, 2 \mathrm{H}, \mathrm{Ar}-\mathrm{H}), 6.83(\mathrm{t}, J=6.0 \mathrm{~Hz}, 1 \mathrm{H}$, $\operatorname{Ar}-\mathrm{H}$ ), 6.11 (d, J= $\left.15.0 \mathrm{~Hz}, 2 \mathrm{H}, \mathrm{CH}_{\text {alkene }}\right), 5.50$ (br s, $\left.4 \mathrm{H}, \mathrm{N}-\mathrm{CH}_{2}\right), 2.64-2.56\left(\mathrm{~m}, 4 \mathrm{H}, \mathrm{CH}_{2}\right), 1.78$ (quin, J=6.0 Hz, 2H, CH $)_{2}, 1.65\left(\mathrm{~s}, 12 \mathrm{H}, \mathrm{CH}_{3}\right.$ ). IR (ATR) 2924, 1592, 1433, 1345, 1271, 1093, 886, $664 \mathrm{~cm}^{-1}$. MS (ESI) m/z: $792[\mathrm{M}]^{+}$. HRMS(FAB): Calcd for $\mathrm{C}_{58} \mathrm{H}_{54} \mathrm{~N}_{3}[\mathrm{M}]^{+}$792.4312, found 792.4307. $\lambda_{\max }=769$ nm. m.p. $214-216^{\circ} \mathrm{C}$.

\title{
Results and Discussion
}

The synthesis of both the rigid (2a-j - 3a-j) and linear (4a-j) cyanine dyes was straightforward and required no harsh or unusual synthetic methodologies. ${ }^{7-8}$ The majority of the mesoaminophenyl substituted heptamethine dyes presented in this manuscript are novel and have been analysed by NMR as well as HMRS. The salts of 2,3,3-trimethylindolenine (1a-e) and 1,1,2-trimethyl- $1 \mathrm{H}$-benzo[e]indole (1f-j) were readily prepared via an $\mathrm{N}$-alkylation with the corresponding alkyl/benzyl halides or 1,4-butanesultone. ${ }^{9}$ In order to synthesise the rigid cyanine dyes (2a-j and 3a-j), a Vilsmeier-Haack formylation of cyclohexanone followed by reaction with aniline produced the reactive imine (VHI) in $97 \%$ yield. This was followed by an aldol-like condensation of the $\mathrm{N}$-alkylated salt precursors with this imine under basic conditions. Upon purification via column chromatography, the rigid meso-chloro substituted dyes (2a-j) were isolated in moderate to low yields. Refluxing (2a-j) in ten equivalents of aniline in anhydrous DMF yielded the crude rigid meso-aminophenyl substituted dyes (3a-j) which were purified again by column chromatography to give the target compounds, also in moderate to low yields. The synthetic route to these dyes is shown in Scheme 1. 
i. $\mathrm{POCl} 3 / \mathrm{DMF}$; ii. Aniline/EtOH/HCl (aq); iii. 1 (a-i)/EtOH/NaOAc; iv. Aniline/DMF/ $/ 2 \mathrm{CO}_{3}$

(a-e) - 2,3,3-trimethylindolenine subunit.

(f-i) - 1,1,2-trimethyl-1H-benzole]indole subunit.

$$
\begin{aligned}
\mathrm{R}=\mathrm{a} / \mathrm{f} & =\mathrm{Me} \\
\mathrm{b} / \mathrm{q} & =\mathrm{Et} \\
\mathrm{c} / \mathrm{h} & =n-\mathrm{Pr} \\
\mathrm{d} / \mathrm{i} & =\mathrm{CH}\left(\mathrm{CH}_{2}\right)_{3} \mathrm{SO}_{3}- \\
\mathrm{e} / \mathrm{i} & =\mathrm{Bn}
\end{aligned}
$$
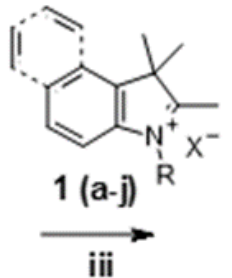

Scheme 1: Route to the heptamethine dyes 3 (a-j). Commercial dyes: IR-746 = 2d; IR-820 = 2i; IR-783 = 4d; ICG = 4i. Note $X$ for compounds a-c and $f-h$ are iodide salts, $e / j$ are bromide salts and $d / I$ are charge balanced inner salts. Purification of the final dyes was accomplished via column chromatography on silica gel eluting with the solvent blends listed within the experimental section as well as the supporting information.

The linear cyanine dyes (4a-j) were produced as shown in Scheme 2, via an in-situ cascade reaction, via the ring opening of $\mathrm{N}$-(2,4-dinitrophenyl)-pyridinium chloride with aniline to produce 5 -anilino- $N$-phenyl-2,4-pentadienylideniminium chloride. This was immediately followed by the direct substitution of the aniline subunit with the $N$-alkylated substituted indolene salt under basic conditions with the whole reaction taking place at room temperature over a period of 12 hours. ${ }^{5}$ The crude dyes were purified via column chromatography using silica gel to obtain the dyes. For all dyes, NMR confirmed their structure and it was noted from the NMR spectra that the dyes were free from solvent and other impurities with high-resolution mass spectrometry confirming the molecular weight.

The photophysical properties of both the linear and rigid NIR heptamethine cyanine dyes used in this study are summarised in Table 1, all in methanol solution. ICG was used as the quantum yield standard producing a value of 0.13 in DMSO. The absorbance and fluorescence spectra of each of the dyes were measured sequentially to reduce photobleaching and solubility issues. 


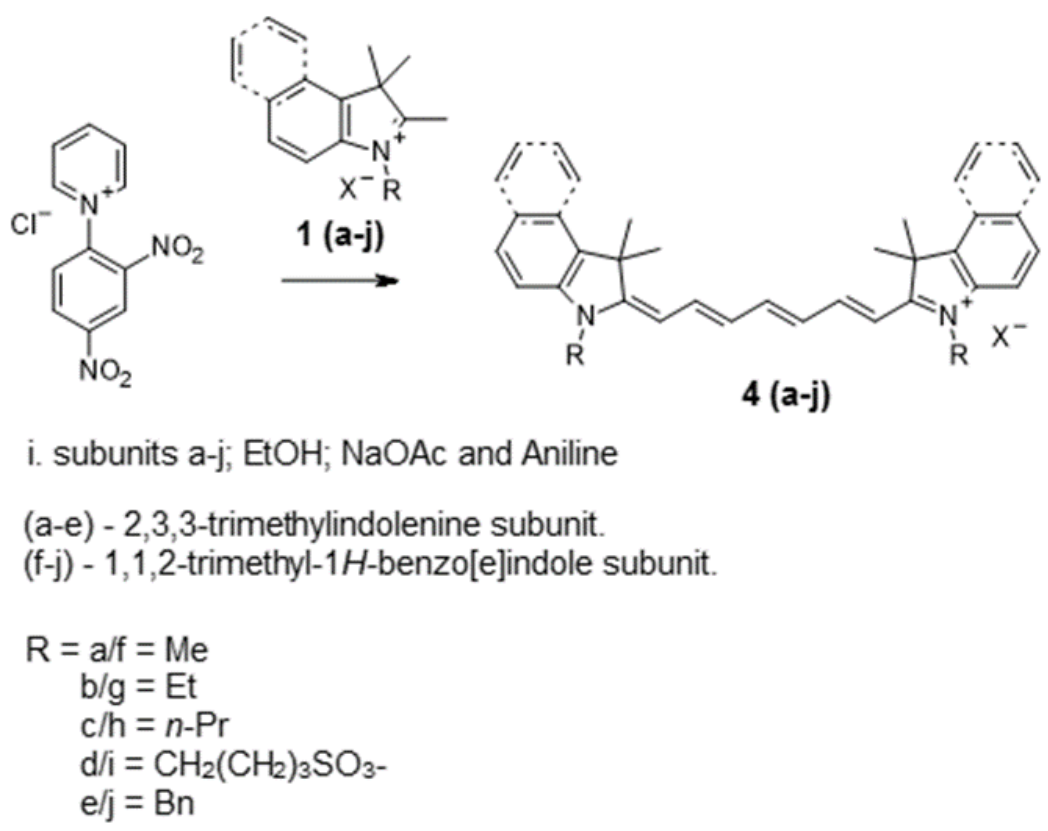

Scheme 2: Route to the heptamethine dyes 4 (a-j). Note $X$ for compounds a-c and $f-h$ are iodide salts, $e / j$ are bromide salts and $d / I$ are charge balanced inner salts. Purification of the final dyes were accomplished via column chromatography on silica gel, eluting with the solvent blends listed within the supporting information.

The fluorescence quantum yields of the dyes were calculated using the relative method, i.e. from plots for standard and cyanine dyes of their individual integrated fluorescence peak areas versus fraction of light absorbed at the excitation wavelength. ${ }^{10-11}$ In this study, emphasis was focused on the effects of structural diversity of the synthesised NIR heptamethine cyanine dyes on their photophysical properties. Firstly, the effect of substituting the meso-chlorine (2a-j) atom with an aminophenyl (3a-j) moiety was investigated, results indicating hypsochromic shifts in absorption wavelength when compared to the meso-chloro analogue. The dyes bearing the phenylamino- moiety exhibited larger Stokes shifts, ranging from $38-58 \mathrm{~nm}$ in comparison to the parent meso-chloro dye, indicating a large structural change between the ground and excited singlet states for these examples. The difference in Stokes shift could be attributed to the nitrogen atom within the aniline participating in an excited-state intramolecular charge transfer (ICT). ${ }^{12}$ One notable drawback with most of the established heptamethine probes is their small Stokes shifts. It is well known that increased Stokes shifts play a key role in avoiding self-quenching and excitation light scattering, thereby extending the potential applications of the current examples as contrast agents. ${ }^{13}$ It was also noted that the relative fluorescence quantum yields of some of the mesoaminophenyl dyes with the greatest Stokes shifts have quantum yields comparable to ICG. It is interesting to note that the largest Stokes shift was observed in both $\mathbf{3 e}$ and $\mathbf{3} \mathbf{j}$ where the substituent is a benzyl group and this may be due to an effect of coplanarity of the core indole rings. 


\begin{tabular}{|c|c|c|c|c|c|}
\hline \multicolumn{3}{|c|}{ Compounds } & \multicolumn{3}{|c|}{ Fluorescence Studies } \\
\hline Code & $\mathbf{R}$ & $\begin{array}{l}\text { Absorption } \\
\text { (nm) }\end{array}$ & $\begin{array}{l}\text { Emission } \\
(\mathrm{nm})^{* 1}\end{array}$ & $\begin{array}{l}\text { Stokes Shift } \\
(\mathrm{nm})\end{array}$ & $\begin{array}{c}\text { Relative } \\
\text { Fluorescence } \\
\text { Quantum Yield*2 }\end{array}$ \\
\hline $2 a$ & Me & 775 & 793 & 18 & 0.073 \\
\hline $2 b$ & Et & 777 & 796 & 19 & 0.080 \\
\hline $2 c$ & $\mathrm{n}-\mathrm{Pr}$ & 781 & 798 & 17 & 0.074 \\
\hline 2d/IR783 & $\mathrm{CH}_{2}\left(\mathrm{CH}_{2}\right)_{3} \mathrm{SO}_{3}^{-}$ & 782 & 802 & 20 & 0.085 \\
\hline $2 e$ & $\mathrm{Bn}$ & 775 & 793 & 18 & 0.084 \\
\hline $2 f$ & Me & 813 & 834 & 21 & 0.028 \\
\hline $2 \mathrm{~g}$ & Et & 815 & 833 & 18 & 0.035 \\
\hline $2 \mathrm{~h}$ & $\mathrm{n}-\mathrm{Pr}$ & 818 & 838 & 20 & 0.029 \\
\hline $2 i / / R 820$ & $\mathrm{CH}_{2}\left(\mathrm{CH}_{2}\right)_{3} \mathrm{SO}_{3}^{-}$ & 820 & 840 & 20 & 0.032 \\
\hline $2 j$ & $\mathrm{Bn}$ & 822 & 845 & 23 & 0.029 \\
\hline $3 a$ & Me & 736 & 786 & 50 & 0.030 \\
\hline $3 b$ & Et & 735 & 786 & 51 & 0.027 \\
\hline $3 c$ & $\mathrm{n}-\mathrm{Pr}$ & 746 & 795 & 49 & 0.054 \\
\hline $3 d$ & $\mathrm{CH}_{2}\left(\mathrm{CH}_{2}\right)_{3} \mathrm{SO}_{3}^{-}$ & 745 & 791 & 46 & 0.047 \\
\hline $3 e$ & $\mathrm{Bn}$ & 736 & 794 & 58 & 0.015 \\
\hline $3 f$ & $\mathrm{Me}$ & 780 & 818 & 38 & 0.049 \\
\hline $3 g$ & Et & 776 & 820 & 44 & 0.062 \\
\hline $3 h$ & $n-P r$ & 778 & 823 & 45 & 0.063 \\
\hline $3 \mathbf{i}$ & $\mathrm{CH}_{2}\left(\mathrm{CH}_{2}\right)_{3} \mathrm{SO}_{3}^{-}$ & 779 & 824 & 45 & 0.067 \\
\hline $3 \mathbf{j}$ & $\mathrm{Bn}$ & 769 & 826 & 57 & 0.048 \\
\hline $4 a$ & $\mathrm{Me}$ & 740 & 770 & 30 & 0.09 \\
\hline $4 b$ & Et & 742 & 770 & 28 & 0.11 \\
\hline $4 c$ & $n-P r$ & 746 & 776 & 30 & 0.13 \\
\hline 4d/IR746 & $\mathrm{CH}_{2}\left(\mathrm{CH}_{2}\right)_{3} \mathrm{SO}_{3}^{-}$ & 747 & 775 & 28 & 0.13 \\
\hline $4 \mathrm{e}$ & $\mathrm{Bn}$ & 748 & 776 & 28 & 0.15 \\
\hline $4 f$ & $\mathrm{Me}$ & 778 & 807 & 29 & 0.064 \\
\hline $4 \mathrm{~g}$ & Et & 781 & 809 & 28 & 0.072 \\
\hline $4 h$ & $n-P r$ & 782 & 811 & 29 & 0.071 \\
\hline $4 i / I C G$ & $\mathrm{CH}_{2}\left(\mathrm{CH}_{2}\right)_{3} \mathrm{SO}_{3}^{-}$ & 784 & 813 & 29 & 0.071 \\
\hline $4 j$ & $\mathrm{Bn}$ & 786 & 814 & 28 & 0.076 \\
\hline
\end{tabular}

Table 1: Photophysical data for dyes within methanol solution. ${ }^{* 1}$ Excitation at $785 \mathrm{~nm} .{ }^{* 2}$ Quantum yields $\pm 10 \%, \lambda_{\max } \pm 1 \mathrm{~nm}$. Relative to ICG.

Although research has been published concerning the addition of heteroatoms directly to the meso- position on the rigid heptamethine dyes, it is noteworthy that there are few examples 
which show the attachment of aromatic amine systems such as aniline. Indeed, Ma et al. have reported the direct attachment of pyridin-4-ol and pyridin-4-thiol directly to the mesoposition of IR-786, through the alcohol and thiol groups respectively, both giving marginal Stokes shifts of 13 and $21 \mathrm{~nm}$ respectively, ${ }^{13}$ i.e. much less than those described here. Other publications have focused on adding a small range of differently-substituted anilines to a rigid heptamethine zwitterionic fluorophore with varying Stokes shifts being identified. ${ }^{14-15}$

The effect of an extra fused benzene on the heterocyclic system was also investigated and data indicated that the dyes with the extra benzo[ $e]$-fusion exhibited longer absorption and emission wavelengths than the indolium-based dyes. It is recognised that the increase in the conjugation of the chromophore system also increases the wavelengths of NIR dyes along with the planarity of the dyes. ${ }^{16}$

\section{Conclusions}

The small library of rigid meso-aminophenyl substituted dyes prepared exhibited a 1.5 - 2 fold increase in Stokes shift when compared against meso-chloro or linear analogues. Furthermore, compound 3i demonstrated a 2-fold increase in relative fluorescence quantum yield compared to the structural standard dye IR820, whilst a greater than 2-fold increase was seen in its Stokes shift. Based on their photophyscial properties, further development and investigation of amino-substituted hepthamethine dyes is clearly of importance to the contrast agent field.

\section{Conflicts of interest}

There are no conflicts to declare.

\section{Acknowledgements}

We are pleased to acknowledge the financial support from the Faculty of Science and Technology, University of Central Lancashire.

\section{References and Notes}

1. S. Luo, E. Zhang, Y. Su, T. Cheng, C. Shi, Biomaterials., 32 (2011) 7127.

2. J. O. Escobedo, O. Rusin, S. Lim, R. M. Strongin, Curr Opin Chem Biol., 14 (2010) 64.

3. E. A. Owens, M. Henary, G. El Fakhri, H. S. Choi, Acc Chem Res., 49 (2016) 1731.

4. E. A. Te Velde, T. Veerman, V. Subramaniam, T. Ruers, Eur J Surg Oncol., 36 (2010) 6.

5. O. A. Okoh, R. H. Bisby, C. L. Lawrence, C. E. Rolph, R. B. Smith, Journal of Sulfur Chemistry., 35 (2014) 42.

6. F. Song, X. Peng, E. Lu, R. Zhang, X. Chen, B. Song, J. Photochem Photobiol A: Chem., 168 (2004) 53.

7. J. H. Flanagan, S. H. Khan, S. Menchen, S. A. Soper, R. P. Hammer, Bioconj. Chem., 8 (1997) 751.

8. S. E. Steinhardt, C. D. Vanderwal, J. Am. Chem. Soc., 131 (2009) 7546.

9. A. R. Tyler, A. O. Okoh, C. L. Lawrence, V. C. Jones, C. Moffatt, R. B. Smith, Eur. J. Med. Chem., 64 (2013) 222. 
10. G. Chapman, M. Henary, G. Patonay, Anal. Chem. Insights., 6 (2011) 29.

11. M. J. Kosch, S. Mäkinen, F. Sigernes, O. Harang, F. Sigernes, D. A. Lorentzen, 30th Annual European Meeting on Atmospheric Studies by Optical Methods, (2003) 50.

12. X. Peng, F. Song, E. Lu, Y. Wang, W. Zhou, J. Fan, Y. Gao, J. Am. Chem. Soc., 127 (2005) 4170.

13. X. Ma, M. Laramie, M. Henary, Bioorganic Med. Chem. Lett., 28 (2018) 509.

14. H. Lee, J. C. Mason, S. Achilefu, J. Org. Chem., 73 (2008) 723.

15. C. N. Njiojob, E. A. Owens, L. Narayana, H. Hyun, H. S. Choi, M. Henary, J. Med. Chem., 58 (2015) 2845.

16. M. Sauer, J. Hofkens, J. Enderlein, Handbook of Fluorescence Spectroscopy and Imaging: From Single Molecules to Ensembles. 2011 Wiley-VCH Verlag GmbH \& Co. KGaA. 